\section{Buchrezension zu: Membranes to Molecular Machines}

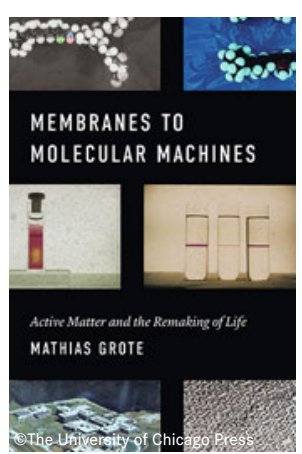

Membranes to Molecular Machines

Active Matter and the

Remaking of Life

Mathias Grote

286 S., The University of Chicago Press, 2019. $H C, 42,33 €$

ISBN: 9780226625157

Auch als PDF erhältlich

DOI: $10.1007 / \mathrm{s} 12268-020-1386-2$

(C) Der Autor 2020

Es ist nicht nur zeitlich ein langer Weg von Friedrich Köhlers Harnstoffsynthese - die die Vitalismus-Theorie trotzdem nicht beendete - bis zu dem neuen (alten) Forschungsgebiet der Synthetischen Biologie. Noch in den 70er-Jahren des letzten Jahrhunderts waren teleologische Ideen weiterhin präsent, wie beispielsweise in Jaques Monods Zufall und Notwendigkeit diskutiert.

Mathias Grote hat nun in seinem bemerkenswerten Buch über Membranen und molekulare Maschinen überzeugend dargelegt, dass biologische Prozesse allein aus den Eigenschaften inrer Komponenten und Strukturen erklärt werden können. Als Fallbeispiel beschreibt er detailliert die Entdeckung und Bedeutung des Membranproteins Bakteriorhodopsin nicht nur anhand der Literatur, sondern auch nach persönlichen Interviews mit wichtigen Protagonisten dieses so wichtigen Forschungsgebietes. Dieses relativ einfache Membranprotein bietet entscheidende Vorteile, die die Forschung und
Methodenentwicklung nicht nur auf dem speziellen Gebiet dieser Licht-getriebenen Protonenpumpe vorantrieben, sondern auch andere Forschungsgebiete entscheidend beeinflussten. So wurden z. B. Infrarot- und Kernspinresonanz(NMR)-Spektroskopie in der Analyse von Bakteriorhodopsin eingesetzt und weiterentwickelt. Entscheidend sind auch die elektronenmikroskopischen Untersuchungen um N. Unwin und R. Henderson, die ein neues Verständnis von Membranen und Membranproteinen ermöglichten.

Auch weitere beschriebene Durchbrüche machen das Buch von Mathias Grote so lesenswert und unterhaltsam. Die ersten Beobachtungen von D. Oesterhelt über die Purpurmembran sind ein sehr schönes Beispiel dafür, dass "Beware of the Unaware" zu neuen Erkenntnissen führt, die über das Erwartete herausgehen. Die Arbeit von E. Racker und W. Stoeckenius über die Licht-getriebene Synthese von ATP in Vesikeln, die Bakteriorhodopsin und ATP-Synthase enthalten, war nicht nur für die chemiosmotische Theorie von P. D. Mitchell entscheidend. Diese Experimente waren auch ein sehr frühes Beispiel für das Gebiet der Synthetischen Biologie.

Mathias Grote hat in seinem Buch von den Anfängen der Membranbiologie bis zur Entwicklung von Biochips einen großen inhaltlichen und philosophischen Bogen gespannt. Es ist nicht nur für Studierende und angehende Wissenschaftlerinnen und Wissenschaftler geeignet, sondern bietet auch für Lehrende eine exemplarische Quelle von Informationen über die Anfänge eines kleinen Forschungsgebietes, das später zu neuen unvorhergesehenen methodischen und konzeptionellen Entwicklungen geführt hat.

Martin Engelhard,

Max-Planck-Institut für molekulare Physiologie, Dortmund, martin.engelhard@mpi-dortmund.

\section{mpg.de}

* Funding Open Access funding provided by Project DEAL.

* Open Access: Dieser Artikel wird unter der Creative Commons Namensnennung 4.0 International Lizenz veröffentlicht, welche die Nutzung, Vervielfältigung, Bearbeitung, Verbreitung und Wiedergabe in jeglichem Medium und Format erlaubt, sofern Sie den/die ursprünglichen Autor(en) und die Quelle orden Link zur Creative Commons Lizenz beifügen und angeben, ob Änderungen vorgenommen wurden. Die in diesem Artikel enthaltenen Bilder und sonstiges Drittmaterial unterliegen ebenfalls der genannten Creative Commons Lizenz, sofern sich aus der Abbildungslegende nichts anderes ergibt. Sofern das betreffende Material nicht unter der genannten Creative Commons Lizenz steht und die betreffende Handlung nicht nach gesetzlichen Vorschriften erlaubt ist, ist für die oben aufgeführten Weiterverwendungen des Materials die Einwilligung des jeweiligen Rechteinhabers einzuholen. Weitere Details zur Lizenz entnehmen Sie bitte der Lizenzinformation auf http://creativecommons.org/ licenses/by/4.0/deed.de. 